\title{
ADVANCED CONTROL SYSTEMS IN TWO WHEELER AND FINDING THE COLLISION SITE OF THE VEHICLE USING GSM
}

\author{
P. Pandiaraj ${ }^{1}$, P. Kalaiselvan ${ }^{2}$ \\ ${ }^{1}$ Assistant Professor, Department of ECE, Hindustan Institute of Technology and Science, Hindustan University, OMR \\ Padur, Chennai-603103, India. \\ ${ }^{2}$ Student- M. Tech Embedded system, Department of ECE, Hindustan Institute of Technology and Science, Hindustan \\ University, OMR Padur, Chennai-603103, India.
}

\begin{abstract}
In the earlier days, vehicles such as Motorbikes hardly had Mechanical support in all concerns, in the recent past the development in Electronic devices in Motorbikes are evolving, with components such as E-breaks, Digital speedometers, etc. and most interesting from an engineering point of view, dealing with motorcycle dynamics is more complex than it is for four wheeled vehicles. In fourwheeled vehicles, electronic stability control (ESC) was introduced in the recent past to improve passengers' safety in critical driving conditions and it is now part of most commercial cars. For two-wheeled vehicles, the design of such a control system is an open problem, and it constitutes quite a challenging task due to the complexity of two-wheeled vehicles dynamics and to the strong interaction between the vehicle and the driver, so to encourage this scheme I am Designing an advanced electronic active stability control system with ABS assistance for e-bikes providing the much needed vehicle roll stability control in situations such as high speed cornering etc. Designing a system that, in any case of emergency situation such as crashing, will send the bicycle accident location to nearby hospitals/emergency services with the assistance of GSM
\end{abstract}

Keywords- Antilock Breaking System (ABS), Electronic Stability Control (ESC), Degree of freedom (DoF), Micro Electro Mechanical System (MEMS)

\section{INTRODUCTION}

Vehicles in everyone's life play a vital role, more over the safety is considered to be primary of all when thinking of it, According to a statistics, $40 \%$ of the traffic accident occur due to the long braking distance, unbalanced slipping off and braking deviation, and thus the automobile brake efficiency is an important factor for the safety of driving.

In the present days bikes are enhanced with advanced acceleration and breaking systems. One such effective design in the breaks of the two wheeler is the Anti-lock Breaking system (ABS) that induces pressure on the break by making the vehicle to halt with in some fraction of seconds and avoids collision with other vehicles or skidding on the road. This System is already existing in the cars before introducing to bikes and had a lot more effect on the breaking control and another feature was also available with the ABS that provides Active stability control using a device known to be the Electronic Stability Control (ESC) but this system was not introduced in bikes, as it constitutes quite a challenging task due to the complexity of two wheeled vehicles

Dynamics and to the strong interaction between the vehicle and the driver. Since in India the usages of two wheelers are more compared to four wheelers, they require more attention as far as safety is concerned.

A reasonable solution for solving this issue is by designing an innovative control Architecture which allows us to enhance the active stability and safety of the vehicle guaranteeing a good driving feeling.

\section{OVERVIEW OF ABS AND ESC}

One important safety technology used in high end cars and trucks is called ABS (Anti-Lock Braking System) which has already become a mandatory feature for vehicles in US and Europe. As a matter of fact, it is an electro-mechanical system that helps reduce wheel skid in automobiles by controlling the brake force applied to each wheel, making it easier to stay in control while riding. ABS equipped bikes are still evolving.

Active Stability Control or Electronic Stability Control (ESC) is a safety feature that improves the vehicle stability by sensing yaw rate and roll rate in order to improve steering control in four-wheeled vehicles. It was introduced to improve passenger's safety in critical driving conditions such as cornering or sudden obstacle avoiding manure. This system uses the ABS at its heart for its longitudinal braking control and senses vehicle dynamics and drivers intention in order to 
stabilize the vehicle by avoiding rolling, skidding and loss of traction.

ABS equipped bikes are still evolving. The figure below shows bike fitted with ABS.

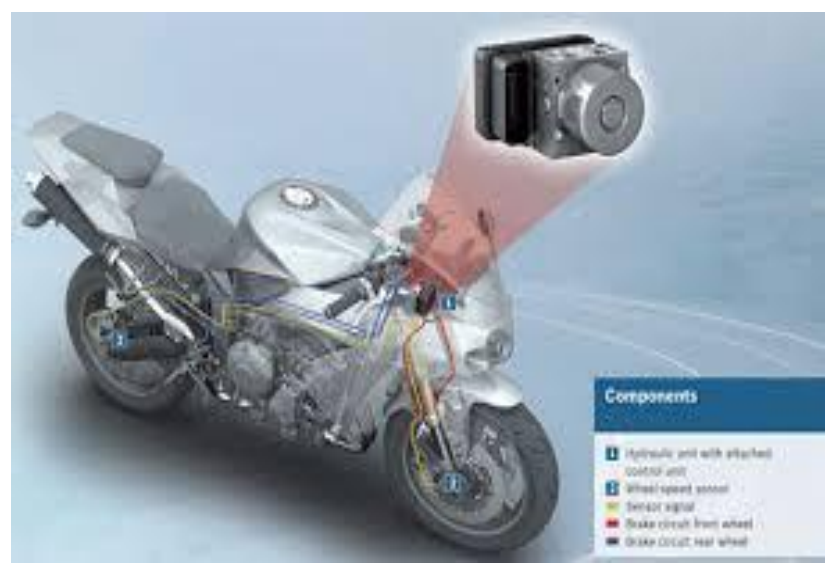

Fig 1 Bike with ABS Equipped

Electronic Stability Control (ESC) helps drivers to avoid crashes by reducing the danger of skidding, or losing control as a result of over-steering. ESC becomes active when a driver loses control of their car. It uses computer controlled technology to apply individual brakes and help bring the car safely back on track, without the danger of fish-tailing. According to the Information from a source, ESC technology is being adopted rapidly by Australian manufacturers and importers - particularly as Victoria has mandated that all new cars registered from January 2011 must be fitted with ESC. This mandate came into effect almost one year ahead of the rest of Australia and also Europe and America. Fitment of ESC has grown enormously since 2004, with the fitment rate increasing from around $12 \%$ in 2004 to $76 \%$ in early 2011 . The highest rate of ESC fitment is in Sweden with a fitment rate of around $96 \%$

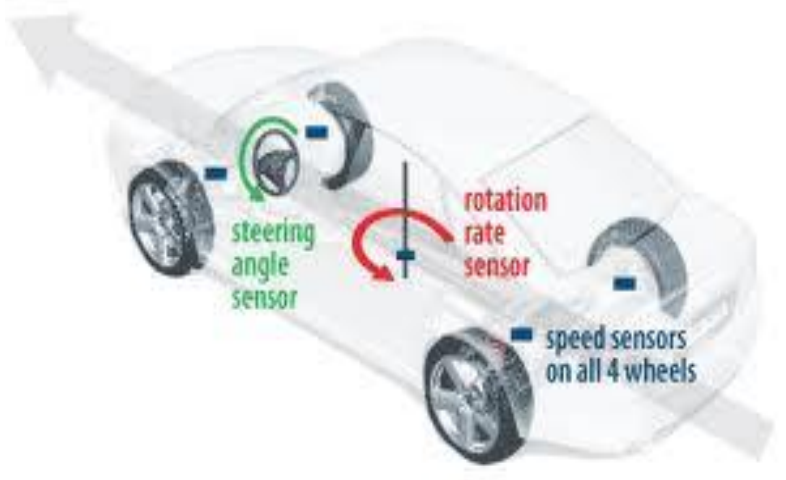

Fig 2 ESC in Four wheeler (Car)
The parts used in bike ABS and car ESC are almost the same, but changes lot in their corresponding working with wheels and dynamic control units as ESC requires more control systems. The Electronic Stability Control ESC integrates ABS and Traction Control System, but has the added feature of a yaw torque control, a functionality that prevents skidding. It is designed to help drivers maintain control of their vehicles in sudden manoeuvres such as rapid steering and counter steering, sudden lane changes and obstacle-avoidance manoeuvres. ESC is always active. 25 times a second, it compares whether the driver's steering input is corresponding to the actual direction in which the vehicle is moves. If the vehicle moves in a different direction, either understeering or over steering, it detects the critical situation and reacts immediately. To do this, the vehicle's braking system is used as a tool for controlling the vehicle back to its track. Specific braking intervention is directed at individual wheels, such as the inner rear wheel to counter understeer, or the outer front wheel during over steer. This control will give a strong interaction on selective braking interventions that generates the desired counter force, so that the car reacts easily as the driver intends. ESC not only initiates braking control, but can also manage it on the engine side to accelerate the driven wheels. ESC substantially reduces the complexity of the steering process and lows down the demands placed on the driver the rate at which the ESC installation is hiked in terms of percentage/year since 2006 is monitored as such.

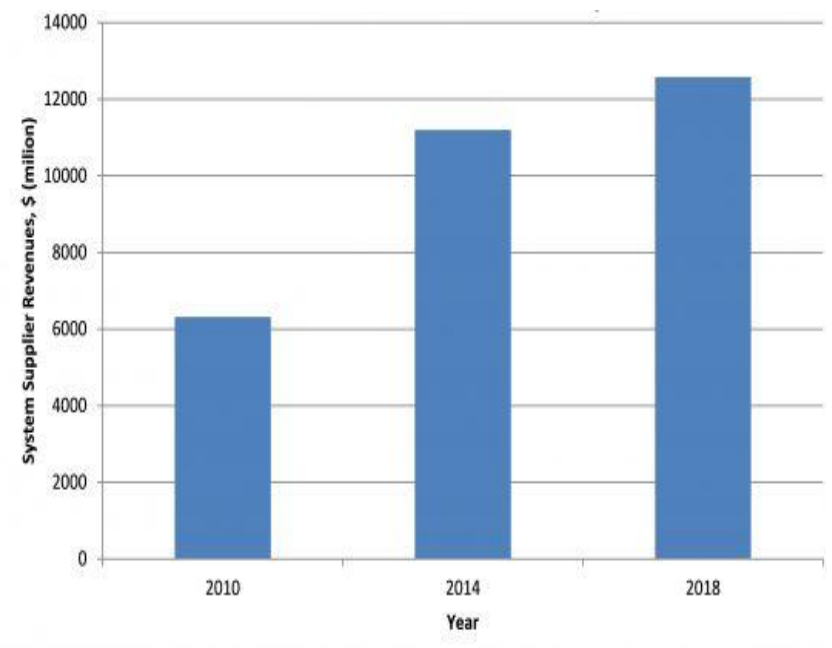

Fig .3 World Market for ABS and ESC systems.

\section{CIRCUIT DESCRIPTION OF BIKE}

The design of stability motorbike comprises of three different Circuit Units and their corresponding circuit description. Antilock Breaking System alone cannot have the full impact on the safety divine, it needs the support of Electronic stability Control to access throughout the drive with long breaking and bear with deadly curves which creates tragic steering such as understeering and over steering. The location of a stationary 
vehicle or that which is in movement can be identified without the help of GPS too, which reduces the burden of spending more penny compared to this small and simple device known to be the Digital MEMS 6-DOF magnetometer which is highly used in vehicles with stability Control, which makes emergency calls/alarms in case of Roll over detection, crush detection, anti-theft.

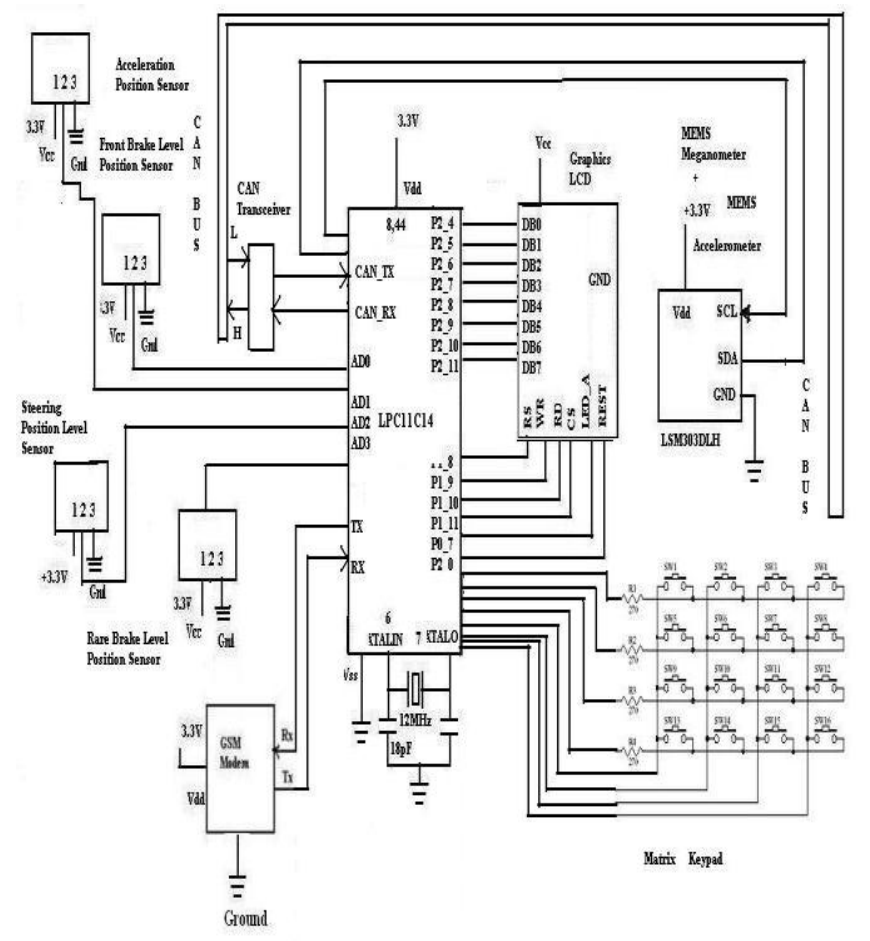

Fig. 4 Dashboard circuit with ABS and ESC

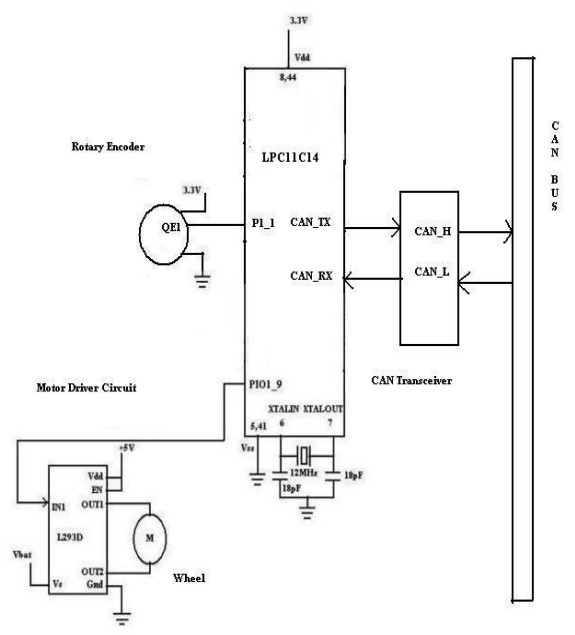

Fig.5 Front wheel Unit

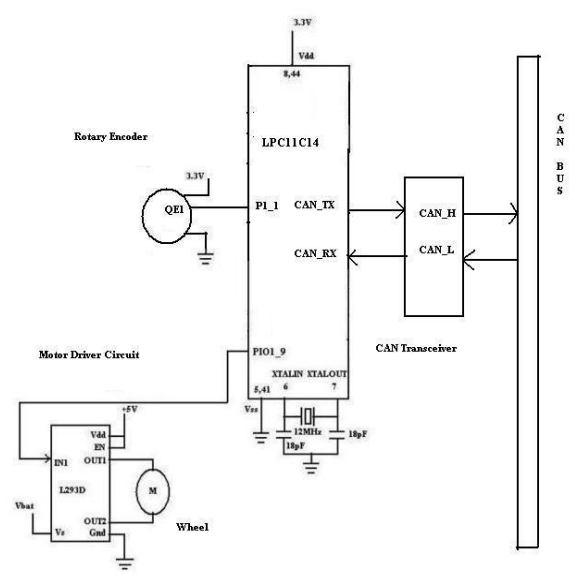

Fig.6 Rear wheel Unit.

\section{COMPONENTS DESCRIPTION}

\subsection{LPC 11 CO0}

The LPC1100 is the world's first Cortex-M0 based microcontroller series offering users a cost effective, very easy to use 32-bit MCU which is code and tool compatible with other NXP ARM based MCU products. With 32-bit performance combined with multiple power modes and very low Deep sleep power, the LPC11xx offers industry leading energy efficiency greatly extending battery life. The LPC11xx sets new benchmarks in performance efficiency with dramatically improved code density enabling longer battery life and lower system costs. It has some additional features such as Serial Wire Debug and Serial Wire Trace Port, Highcurrent output driver $(20 \mathrm{~mA})$ on one pin, High-current sink drivers $(20 \mathrm{~mA})$ on two pins, Integrated PMU (Power Management Unit) to minimize power consumption during Sleep, Deep-sleep, and Deep power down modes, Single $3.3 \mathrm{~V}$ power supply (1.8 V to $3.6 \mathrm{~V}), 15$ GPIO pins can be used as edge and level sensitive interrupt sources, Cock generation unit with divider that can reflect the main oscillator clock, IRC clock, CPU clock, and Watchdog clock, Processor wake-up from Deep-sleep mode via interrupts from various peripherals, Power-On Reset (POR), Crystal oscillator with an operating range of $1 \mathrm{MHz}$ to $25 \mathrm{MHz}$, PLL allows CPU operation up to the maximum CPU rate without the need for a high-frequency crystal. May be run from the main oscillator, the internal RC oscillator, or the Watchdog oscillator, Available as 48-pin LQFP package and 33-pin HVQFN

\subsection{CAN Controller MCP2515}

Microchip Technology's MCP2515 is a stand-alone Controller Area Network (CAN) controller that implements the CAN specification, version 2.0B. It is capable of transmitting and receiving both standard and extended data and remote frames. 
The MCP2515 has two acceptance masks and six acceptance filters that are used to filter out unwanted messages, thereby reducing the host MCUs overhead. The MCP2515 interfaces with microcontrollers (MCUs) via an industry standard Serial Peripheral Interface (SPI).

\subsection{Graphics LCD PCD8544}

The PCD8544 is a low power CMOS LCD controller/driver, designed to drive a graphic display of 48 rows and 84 columns. All necessary functions for the display are provided in a single chip, including on-chip generation of LCD supply and bias voltages, resulting in a minimum of external components and low power consumption. The PCD8544 interfaces to microcontrollers through a serial bus interface. The PCD8544 is manufactured in n-well CMOS technology.

\subsection{MEMS Sensor LSM303DLH}

The LSM303DLH provides three-dimensional heading accuracy within buildings, automobiles, and at high latitudes in such places as the northern US, Canada, and northern Europe, where the declination angle of the earth's field is difficult to measure with Hall-type sensors. In combination with software drivers for heading, auto calibration, and soft-iron/hard-iron compensation - available for a variety of popular mobile phones' operating systems - the LMS303DLH six-dimensional sensor gives system designers a powerful instrument for implementation of navigation functions. The LSM303DLHC features two data-ready signals (RDY) which indicate when a new set of measured acceleration data and magnetic data are available, therefore simplifying data synchronization in the digital system that uses the device.

\section{SOFTWARE TOOL USED}

\subsection{LPCXpresso IDE}

LPCXpresso is a new, low-cost development platform available from NXP. The software consists of an enhanced, Eclipse-based IDE; LPCXpresso is an end-to-end solution enabling embedded engineers to develop their applications from initial evaluation to final production.

The hardware consists of the LPCXpresso development board which has an LPC-Link debug interface and an LPC ARMbased microcontroller target. The $\mathrm{C}$ programming environment includes professional-level features. The LPCXpresso IDE can build an executable of any size with full code optimization and it supports a download limit of $128 \mathrm{kB}$ after registration. Features are more such as LPCXpresso is a complete tool chain for LPC1000 series of Cortex-M microcontrollers, eclipse based IDE, GNU Compiler, Linker and Libraries, Enhanced GDB Debugger, Supports LPC-Link Programmer and Debugger, Developed by NXP Semiconductors and CodeRed Technologies.

\subsection{Operating System used- Free RTOS}

FreeRTOS is professional grade, license free, robust, open Source Real-Time Kernel, Supports Mutex, Semaphores, Queues and C Configured for both Pre-emptive and Cooperative schedulers, Configured for both Pre-emptive and Cooperative schedulers, Configured for both Pre-emptive and Cooperative schedulers, Ported to Cortex-M0 (LPC1000), Works with LPCXpresso tool chain, Takes less than $4 \mathrm{~KB}$ flash memory

\section{FUNCTIONAL DESCRIPTION}

Multiple sensor inputs are used in the active stability system.to determine the breaking force that needs to be applied as per the corresponding requirement. Using acceleration position level sensor Acceleration is measured, front and rear brakes are measured with brake level position sensor, steering angle is measured by steering wheel position sensor which also calculates the break force along with driver accelerator input including wheel speed. If any situations such as vehicle skid or roll or even wheel lock on both wheels are detected, then the breaking pressure required for both wheels are calculated by the controlled and the pressure is applied differentially using electrical motors, which responds to the rider's lever squeezing force

Supposing if ABS system is active while the driver applies sudden break, then the break pressure is calculated by the controller and applies on both the wheels differentially thus reducing the movement of wheel. The skidding threshold level is set to the rider which allows the Adjustment keypad during system activation, allowing different characteristics performances depending on the trail surface. loss of steering controls such as over steering(more than that required to steer) or understeering (less steering than that required) are detected using the steering position level sensor. If the ESC is active then it will detect both understeering as well as over steering and controls the speed automatically

Using 6-DOF Digital MEMS compass module the location of vehicle is sensed, which as a 3-axis MEMS accelerometer and a 3-axis MEMS magnetometer inbuilt within the module so, the direction without any use of GPS is being measured. In case of sudden crash situation the 3-axis MEMS accelerometer senses it and the system sends an emergency message automatically with the use of GSM to nearest Emergency service or to home with the help of embedded vehicle location data. The message sent will direct the location as well as the time at which the crash is detected.

The system totally consist of three Electronic Control Units(ECU's) interconnected with each other, in which two of them are dedicated for front wheel control and rear wheel control, both the wheels are controlled by using two different DC motors, ECU measures the speed and direction of the wheels interfaced with the rotary encoder. Driver input unit 
sensors such as break level position sensor, acceleration level position sensor and steering wheel position sensor are interfaced with the main dashboard unit which is the third ECU. Other interfaces are also fixed on this ECU such as graphical LCD and GUI are provided which acts as dashboard display and vehicle status monitoring, also the MEMS compass is connected to this unit.

The whole system of three ECUs are connected each other through a closed network called as Controller Area Network (CAN). Each CAN has its own function as it is well known for its event-driven protocol, most often used in the automotive applications. To meet with the real time target and make this system long lasting, Free RTOS is most preferred to run, which is well known open source real-time kernel ever.

\section{ADVANTAGES}

Comparing to the currently available mechanical models, electronic ABS systems are more superior, Digital compass direction sensor provides 360 degree direction by sensing earth's magnetic field. ARM Cortex-M0 microcontroller is used which has an in built CAN controller, Without the need of GPS, the cost of tracking is very less, Since CAN network is used, excess wires are reduced, The real-time kernel being open source is most efficient, which can be ideally used for networking within ECU's, Two wheeled vehicle with active stability control is attempted by designing for the first time.

\section{CONCLUSIONS}

As an overview of this paper E-Bike is designed with Antilock Breaking System and all the necessary sensor inputs for stability maintenance have been projected with corresponding outcomes regarding the rider's comfort. The very basic feature of four wheeler is drastically fulfilled and crash vehicle tracking is done with digital compass by using GSM. In future this can be enhanced in with the use of GPS or Mobile tracking systems or even more evolving technology for more accuracy.

\section{ACKNOWLEDGMENTS}

I am very much Grateful to my parents who supported in all the ways they could, my tutors who supported me to successfully fulfil my paper as it came up with a conclusion of Real time. Also I thank all the staffs in my department, and to all my friends who ideally encouraged me by sharing the virtual ideas.

\section{REFERENCES}

[1]. Rajesh Rajamani, Senior Member, IEEE, and Damrongrit (Neng) Piyabongkarn, Member, IEEE, New Paradigms for the Integration of Yaw Stability and Rollover Prevention Functions in Vehicle Stability Control VOL. 14, NO. 1, MARCH 2013.
[2]. Mohammed H. Al-Mola, M. Mailah, S. Kazi, A.H. Muhaimin, M.Y. Abdullah Faculty of Mechanical Engineering Universiti Teknologi Malaysia, Robust Active Force Controller for an Automotive Brake System 2012 Third International Conference on Intelligent Systems Modelling and Simulation.

[3]. Pier Paolo De Filippi et al, Dipartimento di Elettronica e Informazione, Politecnico di Milano, Milan, 20133 Italy .The Delft Center for Systems and Control, Delft University of Technology, Delft, The Netherlands Preprints of the 18th IFAC World Congress Milano (Italy) August 28 - September 2, 2011. [4]. Y. Chen and J. Wang, "Energy-efficient control allocation with applications on planar motion control of electric ground vehicles," in Proc. Amer. Control Conf., San Francisco, CA, 2011, pp. 2719-2724.

[5]. G. A. Magallan, C. H. D. Angelo, and G. O. Garcia, "Maximization of the traction forces in a 2WD electric vehicle," IEEE Trans. Veh. Technol., vol. 60, no. 2, pp. 369 380, Feb. 2011.

[6]. M. Tanelli, M. Corno, et al, Active braking control of twowheeled vehicles on curves. International Journal of Vehicle Autonomous Systems, 7(3/4):243-269, 2010.

[7]. J. Tjonnas and T. A. Johansen, "Stabilization of automotive vehicles using active steering and adaptive brake control allocation," IEEE Trans. Control Syst. Technol., vol. 18 , no. 3, pp. 545-558, May 2010.

[8]. XU Shengming, The diagnosis and analysis of accidental car $\square$ s antiskid brake system, ABS [M]. ChengDU, The Press of Southwest Jiaotong University, 2009,45,58

[9]. J. Lu, D. Messih, and A. Salib, "Roll rate based stability control-The roll stability control system," presented at the 20th Enhanced Safety Vehicles Conf., Lyon, France, 2007, Paper 07-136

[10]. M. C. Walz, "Trends in the static stability factor of passenger cars, ligh trucks, and vans," Nat. Hwy. Traffic Safety Admin., Washington, DC, Tech. Rep. DOT HS 809 868, Jun. 2005.

\section{BIOGRAPHIES:}

Mr P.Pandiaraj received his Bachelor Degree in Electronics and Communication Engineering from Madurai Kamaraj University and Completed His M.E in the same discipline from Anna University, Chennai. He is working as Assistant Professor in Hindustan Institute of Technology and science (Hindustan University), at Chennai, India. His Area of interest is Wireless, Embedded and Mobile Computing.

P.Kalaiselvan received his Bachelor Degree in Electronics and Communication Engineering from KCG College of technology under Anna University, Chennai during the year 2007-2011 and $\mathrm{He}$ is currently doing his M. Tech in Embedded Systems from Hindustan Institute of Technology and science (Hindustan University), during the year 2012-2014 at Chennai, India. His Area of studies is Embedded, Networking and Mobile Computing 